\title{
A Comparison of Intensive Care Unit Care of Surgical Patients in Teaching and Nonteaching Hospitals
}

SAMIR M. FAKHRY, M.D., JEFFREY L. BUEHRER, M.D., GEORGE F. SHELDON, M.D., and ANTHONY A. MEYER, M.D., PH.D.

\begin{abstract}
Three hundred forty-eight teaching (TH) and 282 nonteaching (NTH) hospitals were surveyed to determine how intensive care unit (ICU) care is delivered to surgical patients and current views on surgical critical care. Teaching hospitals were more likely than NTHs to have a separate surgical ICU (92\% versus $37 \%$ ), a dedicated ICU service/physician (37\% versus $7 \%$ ), and a surgeon as director of the ICU (67\% versus $29 \%)$. All THs and 33\% of NTHs provided 24 hour in-house coverage for the ICU. A majority of respondents preferred a surgeon as ICU director (TH, 85\%; NTH, 67\%) and felt that critical care was an essential part of surgery (THs, 87\%; NTHs, 74\%). Most (THs, 58\%; NTHs, $56 \%$ ) thought that a cooperative effort between the primary service and an ICU service provided better patient care, but only $37 \%$ of THs and $22 \%$ of NTHs provided care with such a system. Many (THs, 45\%; NTHs, 33\%) thought that surgeons are willingly relinquishing ICU care. Surgeons continue to desire responsibility for their patients in the ICU and most prefer ICU service involvement provided by surgeons. This discrepancy between what is practiced and what is desired, along with proposed changes in reimbursement for surgery and the recent definition of critical care as an essential part of surgery, may stimulate greater involvement of surgeons in critical care.
\end{abstract}

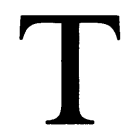

HE CARE OF critically ill patients in the intensive care unit (ICU) is a significant part of modern clinical medicine in all major specialties. Critical care needs account for up to $10 \%$ of hospital beds and up to $15 \%$ of inpatient health expenditures. ${ }^{1-3}$ The increasing complexity in available technology and treatment modalities has placed added demands on practicing surgeons, effectively competing with traditional activities in the operating room, the office, and the wards. Quality patient care requires up-to-date knowledge of critical care and a mechanism to provide this care in a consistent, uninter-

Address reprint requests to Samir M. Fakhry, M.D., Department of Surgery, 167 Burnett-Womack Bldg., CB 7210, University of North Carolina, Chapel Hill, NC 27599-7210.

Accepted for publication August 17, 1990.
From the Department of Surgery, School of Medicine, University of North Carolina at Chapel Hill, Chapel Hill, North Carolina

rupted fashion. The ICU care of surgical patients is delivered in a variety of formats, conforming to the needs and resources of different institutions. The role of surgeons in the care of these critically ill patients has been examined in several recent reports dealing predominantly with the academic setting. ${ }^{4-6}$ These reports stated that surgeons should be responsible for the care of their critically ill patients. The American College of Surgeons, the American Board of Surgery (ABS), and the Residency Review Committee are among the professional organizations that stress the importance of the 'complete care of critically ill patients with underlying surgical problems' to include the ICU stay. The ABS considers critical care among the 'essentials' of general surgery ${ }^{7}$ and has issued a Certificate of Added Qualification in Surgical Critical Care.

In a recent survey of the 296 program directors of approved General Surgery training programs, $91 \%$ thought that critical care should be considered one of the 'essentials' of General Surgery and $72 \%$ of program directors thought that there should be a separate surgical ICU rotation for general surgery residents. ${ }^{8}$ In only $47 \%$ of the programs, however, did a designated surgical ICU service exist to provide surgical residents with the necessary training. Although this survey and other reports ${ }^{4-6,8}$ provide some insight into the ICU care of surgical patients in teaching hospitals $(\mathrm{TH})$, there is little data available from nonteaching hospitals (NTH) on the ways in which care of critically ill surgical patients is delivered. Similarly little is known of the views of surgeons at NTHs with respect to surgical critical care (SCC). This study was undertaken to compare ICU care of surgical patients at THs and NTHs and the views of surgical leaders at both types of institutions with regard to SCC. 


\section{Methods}

A surgical critical care questionnaire was sent to the program directors and/or chiefs of surgery in all the hospitals in the 296 general surgery teaching programs listed in the 1987-1988 Directory of Graduate Medical Education Programs. ${ }^{9}$ A second questionnaire was sent 4 months later if there was no response to the first request. A similar questionnaire was addressed to the chief of surgery at 282 NTHs randomly selected from the American Hospital Association's Guide to the Health Care Field. ${ }^{10}$ The questionnaires addressed the program/hospital description; the available hospital and ICU beds; the attending staff; the ICU resources; the conduct of patient care, including coverage and billing; and the views of the program director or chief of surgery with respect to SCC issues. The two questionnaires were nearly identical, the one sent to THs containing additional questions pertaining to resident education and the one sent to the NTHs containing additional questions related to functions of the attending physicians, some of which are assumed by residents in THs. Questions common to both questionnaires were written in an identical manner.

The data were compiled and entered into a microcomputer database for analysis. Answers were expressed as frequencies of replies and rounded to the nearest $1 \%$.

\section{Results}

We received responses from 235 of 296 academic programs containing data from 348 THs. Answers from individual THs were considered separately and served as the denominator for THs in the analysis. The response rate by general surgery teaching program was $79 \%$ ( 235 of 296). We received responses from 121 NTHs, for a response rate of $39 \%$ (121 of 282 ) (Table 1$)$.

\section{Critical Care Facilities (Table 2)}

The average number of hospital beds at THs was 539 $+/-300$ and at the NTHs it was $338+/-137$. The average number of ICU beds at THs was greater than at NTHs (45 compared to 21), as was the number of surgical ICU beds (17 compared to 9). All hospitals responding had at least one ICU. Ninety-two per cent of THs had a

TABLE 1. Survey Responses

\begin{tabular}{lcc}
\hline \multicolumn{1}{c}{ Surveys } & TH & NTH \\
\hline Surveys sent & 296 programs & 282 hospitals \\
Surveys received & 235 programs & 121 hospitals \\
Response rate & 348 hospitals* & \\
\hline
\end{tabular}

* Some programs had more than one teaching hospital and a survey form was included for each individual hospital.

TH, teaching hospital; NTH, nonteaching hospital.
TABLE 2. Critical Care Facilities

\begin{tabular}{lcc}
\hline \multicolumn{1}{c}{ Facilities } & TH & NTH \\
\hline Average number hospital beds & $539 \pm 300$ & $338 \pm 137$ \\
Average number ICU beds & $45 \pm 28$ & $21 \pm 13$ \\
Average number surgical ICU beds & $17 \pm 14$ & $9 \pm 4$ \\
Hospitals with separate surgical ICU & $92 \%$ & $37 \%$ \\
Most common ICU & Surgical & Combined med- \\
& $(92 \%)$ & surg (69\%) \\
Level 1 Trauma Center designation & $42 \%$ & $6 \%$ \\
Level 2 Trauma Center designation & $22 \%$ & $27 \%$ \\
Level 3 Trauma Center designation & $7 \%$ & $7 \%$ \\
\hline
\end{tabular}

TH, teaching hospital; NTH, nonteaching hospital.

separate surgical ICU while only $37 \%$ of NTHs had a separate surgical ICU. The most common type of ICU at the THs was the surgical ICU, followed by coronary care units (CCU) in $81 \%$, medical ICU (MICU) in $80 \%$, neonatal ICU in $56 \%$, and cardiothoracic ICU in $50 \%$. The most common ICU at the NTHs was the combined medical-surgical ICU, present in $69 \%$ of NTHs, followed by the CCU present in $28 \%$, the SICU in $37 \%$, and the MICU in $33 \%$. Analysis of trauma center designation also was included. Significantly more THs than NTHs had level 1 designation: 135 (39\%) THs compared to 7 (5.8\%) NTHs.

\section{Administration (Table 3)}

A surgeon was the director or codirector of the surgical ICU in $67 \%$ of THs and $40 \%$ (93) of these surgeon directors held an ABS Certificate of Added Qualification in Surgical Critical Care. A surgeon served as director or codirector of the ICU in which surgical patients received care in $29 \%$ of NTHs and only $12 \%$ (4) of these surgeons had the ABS Certificate of Added Qualification in Surgical Critical Care.

\section{Patient Care (Table 3)}

A separate surgical ICU service existed in $37 \%$ of THs. Only $7 \%$ of NTHs had a physician whose sole responsibility was in the ICU. In $33 \%$ of NTHs a physician was available on call in the hospital 24 hours a day, while in the remainder, ICU call was from home or by other arrangement. In THs with a designated surgical ICU service, the care of the patient in the ICU was rendered through a cooperative effort between the primary service and the ICU-based service in $63 \%$ of hospitals. In THs without a designated SICU service, the primary surgeon was solely responsible for care in $72 \%$ of hospitals. At NTHs the primary surgeon was responsible for care in $69 \%$ of hospitals, while care was rendered through a cooperative effort between the primary physician and another physician in $22 \%$ of hospitals. There was no instance in our sample of NTHs of an ICU physician/service assuming primary responsibility for a patient's ICU care and only $10 \mathrm{THs}$ 
TABle 3. Administration and Patient Care

\begin{tabular}{lcc}
\hline & TH (\%) & NTH (\%) \\
\hline Surgeon ICU director & 67 & 29 \\
Director has ABS SCC certificate & 40 & 12 \\
Dedicated SICU service/MD & 37 & 7 \\
24 hour physician coverage & 100 & 33 \\
$\quad$ In house & 37 & 7 \\
In ICU & & \\
Primary responsibility for ICU care provided by & 55 & 69 \\
Primary surgeon & 4 & - \\
ICU service/MD & 33 & 2 \\
$\quad$ Cooperative effort & & \\
ICU service/physician participate in & 51 & 36 \\
$\quad$ Ventilator management & 48 & 36 \\
$\quad$ Emergency interventions & & \\
Referring physician participates in postoperative & & \\
$\quad$ care in ICU & N/A & 5 \\
Always & N/A & 28 \\
Frequently & N/A & 43 \\
Occasionally & N/A & 11 \\
Never &
\end{tabular}

TH, teaching hospital; NTH, nonteaching hospital.

provided ICU care to critically ill surgical patients primarily through the ICU service.

In response to the question to the chief of surgery at NTHs, 'How often do nonsurgical physicians in your hospital expect to provide postoperative critical care management of patients they refer for surgery?' the following answers were received: never, $11 \%$; occasionally, $43 \%$; frequently, $28 \%$; always, $5 \%$; no response, $13 \%$.

When a surgical ICU service existed at a TH, it controlled admissions and discharges in $65 \%$ of hospitals and managed ventilators, fluids, and 'drips' in $75 \%$. The surgical ICU service at THs made rounds with the primary service in $67 \%$ of cases and provided in-house resident coverage with attending back-up in $92 \%$ of hospitals. The ICU physician caring for surgical patients at NTHs controlled admissions and discharges in $16 \%$ of hospitals, managed ventilators, fluids, and 'drips' in $20 \%$, and made rounds with the primary service in $7 \%$ of hospitals. Inhouse physicians were available 'on-call' for the ICU in $33 \%$ of NTHs.

\section{Economic Considerations}

The surgical ICU service in THs billed separately for critical care services in $43 \%$ of hospitals with such a service, for individual consultation in $45 \%$, and for procedures in $50 \%$. The physicians designated for the ICU at NTHs in which surgical patients were managed billed for these services at substantially lesser frequency: $10 \%, 15 \%$, and $12 \%$, respectively.

\section{ICU Bed Availability}

A shortage of ICU beds resulted in a limitation being placed on admissions to the ICU and in operative pro- cedures performed in 59\% of THs and in $24 \%$ of NTHs. When ICU admissions had to be limited, among the reasons cited were nursing shortages $(69 \%$ of THs and $77 \%$ of NTHs), bed availability ( $76 \%$ of THs and $95 \%$ of NTHs), and the unwillingness of physicians to transfer noncritically ill patients out of the ICU ( $18 \%$ of THs and $50 \%$ of NTHs).

\section{Views (Table 4)}

Eighty-seven per cent of chiefs of surgery at THs and $74 \%$ of chiefs of surgery at NTHs believed that critical care should be considered one of the essentials of general surgery. Only $3 \%$ at THs and $12 \%$ at NTHs disagreed. Sixty per cent at both THs and NTHs thought a certificate of Added Qualification in Surgical Critical Care should be offered by the ABS. Forty-five per cent of program directors at THs and $33 \%$ of chiefs of surgery at NTHs thought the care of critically ill surgical patients was being willingly relinquished by surgeons. Eighty-five of those at THs would prefer a surgeon as surgical ICU director and $70 \%$ thought he or she should be certified in critical care, while $67 \%$ at NTHs would prefer a surgeon as surgical ICU director and 55\% thought he or she should be certified in critical care. Most respondents in both groups (THs, $57 \%$; NTH, 56\%) thought optimal care in the surgical critical care setting would be rendered by a combination of the primary service/physician and the ICU service/ physician. Most respondents $(71 \%$ in THs and $54 \%$ in NTHs) would encourage general surgery residents to enroll in critical care training fellowships.

\section{Discussion}

Surgeons have traditionally provided the complete spectrum of care to patients with surgical diseases. ${ }^{10-12}$ They have been responsible for many major advances in all aspects of patient care and participated in the development of the ICU concept and many of the subsequent innovations and technologies. ${ }^{13} \mathrm{~A}$ rapid expansion in knowledge of normal and abnormal physiology and an exponential growth in the technologies of health care have resulted in added demands on physicians providing care in the critical care setting. As these innovations and tech-

TABLE 4. Views of Chiefs of Surgery

\begin{tabular}{lll}
\hline \multicolumn{1}{c}{ View } & \multicolumn{1}{c}{ TH (\%) } & NTH (\%) \\
\hline Prefer surgeon SICU director & 85 & 67 \\
SCC essential in general surgery & 87 & 74 \\
Cooperative effort between ICU service and primary & & \\
$\quad$ service provides better patient care & 58 & 56 \\
Best ICU care with cooperative system & 37 & 22 \\
ICU care of surgical patients being willingly & & \\
relinquished & 45 & 33 \\
\hline
\end{tabular}

TH, teaching hospital; NTH, nonteaching hospital. 
nologies become more elaborate and the therapeutic modalities used in the critical care setting continue to increase in complexity, the practicing surgeon finds it more difficult to maintain a level of proficiency to provide quality care to his or her critically ill patients in the ICU. Surgeons are faced with simultaneous demands in the operating room, the wards, and in the laboratory in academic settings. Sometimes surgeons have found it necessary and, in some cases, convenient to seek the assistance of other physicians in providing portions of the preoperative and postoperative care of patients. As is apparent from our survey, it is not uncommon for the referring physician in the NTH to expect to provide preoperative and postoperative care to patients referred for surgery. Patients in the ICU may require expertise not readily available at the level of the referring physician and thus require the addition of another specialist (often in pulmonary medicine) to the physician roster who generates further charges to the patient. Although a shared, cooperative effort for certain complex problems is practical and may be beneficial, completely relinquishing care is clearly undesirable.

This study shows that there are significant differences in the ICU care of surgical patients at THs and NTHs. This includes differences in both management practices and resource allocation. These differences are due, in part, to the THs being on average larger than the NTHs. The ICUs for surgical patients at THs are also larger than their counterparts in NTHs. There is a higher degree of ICU coverage at THs, with physicians available in-house on a 24-hour basis at all THs but in only $33 \%$ of NTHs. A separate surgical ICU service existed at $37 \%$ of THs, while few $(7 \%)$ NTHs had a physician solely designated for the ICU. The quantitative differences are not completely the result of the greater bed capacity of THs because that does not explain the difference in coverage patterns and in direct patient care practices. We assume that the presence of residents at THs accounts for much of the difference in coverage and management practices and that patient acuity also has a significant effect. The surgeon's rapport with the patient's referring physician and other specialists has practical implications at NTHs; our data suggest that in up to $75 \%$ of cases the surgeon is under some pressure to allow others to provide postoperative care. Our data do not allow us to determine the relative contribution of such factors.

Differences in management practices also were noted. A designated surgical ICU service existed at $37 \%$ of THs while only $7 \%$ of NTHs had a physician designated solely for the ICU in which surgical patients were located. A separate surgical ICU existed in $92 \%$ of THs and was the most common type of ICU at the academic institutions. Only $37 \%$ of NTHs had a separate surgical ICU and the most common type of ICU there was the combined medical-surgical unit. This arrangement and the paucity of surgeon-directors at NTH underscores the shortage of surgeons interested and qualified to administer ICUs in the nonacademic setting. It has been suggested that some surgeons may find it more convenient and remunerative to operate while someone else provides ICU care and administration. ${ }^{6,14}$ Of practical significance, there are more than 3000 internists (predominantly pulmonologists) and anesthesiologists holding critical care certificates from their respective board. As of August 1990, the number of surgeons holding a Certificate of Added Qualification in Surgical Critical Care from the ABS is approximately 508. This is the largest number of any specialty except internal medicine.

While the manner in which ICU care for surgical patients is administered differs significantly between THs and NTHs, the views of surgical leaders at both types of institutions are similar (Table 4). The majority of respondents at both THs and NTHs thought that critical care was an essential part of general surgery and that a surgeon should direct the ICU where surgical patients are treated. More than one half the respondents at both THs and NTHs thought that a cooperative effort between the primary surgeon and an ICU service/physician provided optimal care, but only a minority at both types of hospitals provided care with such a system. A significant number of these leaders thought critical care was being willingly relinquished by surgeons.

The views expressed by the respondents are in accord with the frequent assertion that surgeons provide postoperative care for their patients in the critical care setting and that they retain control of their ICUs. ${ }^{4,6,7,10,11,14}$ The manner in which ICU care is presently provided, however, suggests that the implementation of these ideals is less than desired. This is true at both THs and NTHs, although the situation at NTHs is more pronounced. In a study of ICU care of surgical patients in the private practice environment, Trask and Faber ${ }^{15}$ reached similar conclusions. Their survey of the chiefs of surgery and the nurse managers of ICUs at 188 private hospitals revealed that 'surgeons did not have the principal managing role in the ICU in $70 \%$ to $75 \%$ of the hospitals' and that 'surgeons are relinquishing their responsibilities in the direct care of the preoperative and postoperative critically ill patients.' They suggest that there are three main reasons for this: the complexity of critical care, a lack of economic incentives, and professional liability.

Resolution of these issues may be influenced by forces from within organized surgery as well as external factors. On the one hand, surgeons and their professional organizations have begun to respond to the growing need for trained surgeons to direct ICUs. The ABS has asserted that critical care is an essential part of general surgery and provided a mechanism for credentialing qualified surgeons in SCC. Fellowship programs in critical care are offered 
by 93 institutions in the United States. ${ }^{16}$ Of these approximately 22 are based in academic surgical departments. At present, only five are approved by the Residency Review Committee for Surgery. Proposed changes in reimbursement for surgical services, such as the global fee concept, may provide further impetus for change. If procedure fees included postoperative care, the operating surgeon would be expected to provide ICU care and would therefore need to be reasonably proficient in such care whether in THs or NTHs.

The impact of a designated ICU physician on the care of critically ill patients has been studied. Li et al. ${ }^{17}$ reported that the introduction of a designated ICU team resulted in improved ICU and in-hospital outcomes in a community hospital. This was accompanied by a significant increase in the use of invasive monitoring and blood tests and a shorter ICU stay for lower-risk patients. Knaus et al. ${ }^{18}$ reported that the degree of coordination of ICU care significantly influenced its effectiveness. Therefore there is a role for qualified surgeons to direct surgical ICUs, provide on-site availability, and manage resource allocation and quality assurance.

Our data suggest that most chiefs of surgery at both THs and NTHs endorse a system whereby surgeons control their ICUs and provide care for their critically ill patients. The actual mechanisms by which care is delivered suggests that there is a shortage of qualified surgeons to direct ICUs and manage critically ill surgical patients. Furthermore it appears that general surgeons are often unwilling or unable to provide ICU care, particularly at NTHs. In the present reimbursement climate, the incentives and referral patterns, especially at NTHs, favor a lesser level of involvement of surgeons in ICU care. General surgery training programs may influence these trends by establishing SCC services, maintaining control of their ICUs, and sponsoring fellowships in SCC. Such action would result in continued emphasis on the training of general surgeons in SCC and in providing a pool of qual- ified surgeons who are credentialed in SCC to administer and manage surgical ICUs. It is probable that in the absence of such efforts, surgeons may continue to relinquish their role in the ICU.

\section{References}

1. Critical Care Medicine, Consensus Development Conference Summary, National Institutes of Health, 1983, 4(6).

2. Augenstein J, Peterson E. Economic Considerations in Critical Care. In Shoemaker WC, Ayres S, Grenvik A, et al, eds. Textbook of Critical Care, 2nd edition. Philadelphia: WB Saunders, 1989, pp 1466.

3. Grenvik A. The ICU in the modern hospital. In Reis MD, Langrehr D, eds. The ICU, A Cost-Benefit Analysis. New York: Elsevier Science Publishers, 1986, pp 27-37.

4. Machiedo GW, Blackwood JM, Rush BF Jr. Practice of critical care medicine in academic surgical centers. J Surg Res 1981; 30:223228.

5. The surgeon and intensive care. Am Coll Surg Bull 1985; 70:25-26.

6 . Holcroft JW. Responsibility for care of the critically ill surgical patient. J Surg Res 1985, 38:315-318.

7. Statement of the Advisory Council for General Surgery to the Board of Regents of the American College of Surgeons. Am Coll Surg Bull, 1989, 74:6-7.

8. Meyer AA, Fakhry SM, Sheldon GF. Critical care education in general surgery residencies. Surgery, 1989, 106:392-399.

9. Directory of Graduate Medical Education Programs, 1987-1988. Chicago, American Medical Association, 1987.

10. Meyer AA, Trunkey DD. Critical care as an integral part of trauma care. Crit Care Clin 1986; 2:673-681.

11. Walt AJ. The training and role of the surgeon in the intensive care unit. Surg Clin North Am 1985; 65:753-762.

12. Collins JA, Ballinger WF II. The surgical intensive care unit. Surgery 1969; 66:614-619.

13. Hilberman M. The evolution of intensive care units. Crit Care Med 1975; 3:159-165.

14. Trunkey DD, Blaisdell FW. The resurgence of the barber surgeon. Am Coll Surg Bull 1985; 70:14-15.

15. Trask AL, Faber DR. The intensive care unit-Who's in charge? The private practice view. Arch Surg 1990; 125:1105-1108.

16. Fellowship programs in critical care medicine: 1989/1990. Crit Care Med 1989; 17:83-101.

17. Li TCM, Phillips MC, Shaw L, et al. On-site physician staffing in a community hospital intensive care unit. JAMA 1984; 252:20232027.

18. Knaus WA, Draper EA, Wagner DP, Zimmerman JE. An evaluation of outcome from intensive care in major medical centers. Ann Int Medicine, 1986; 104:410-418. 